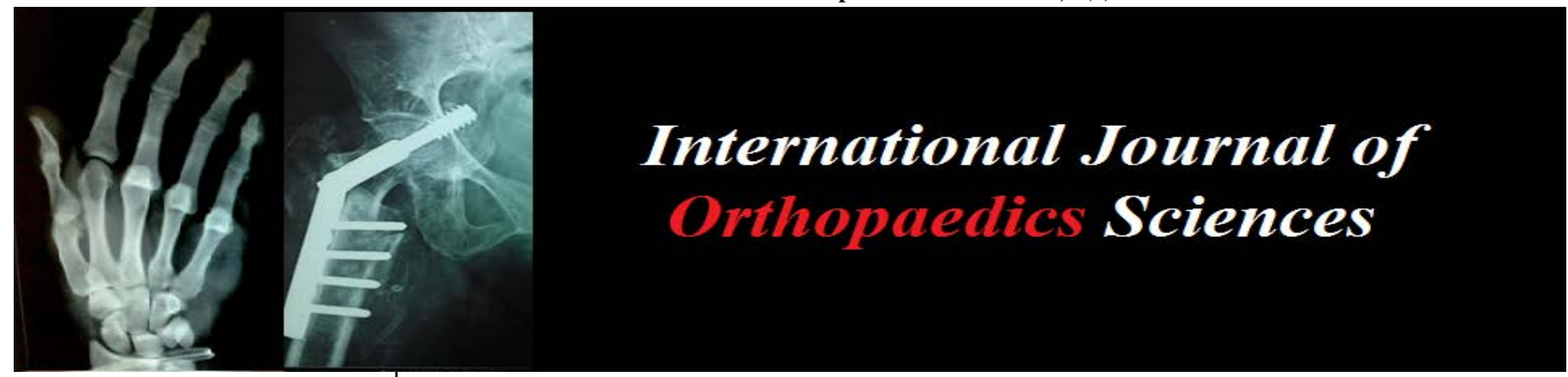

E-ISSN: 2395-1958

P-ISSN: 2706-6630

IJOS 2020; 6(4): 586-591

(C) 2020 IJOS

www.orthopaper.com

Received: 16-07-2020

Accepted: 07-08-2020

\section{Dr. Prabhu S}

Post Graduate, Department of Orthopaedics, Rajah Muthiah Medical College, Chidambaram, Tamil Nadu, India

Dr. Neelakrishnan $R$

Professor and Head, Department of Orthopaedics, Rajah Muthiah

Medical College, Chidambaram,

Tamil Nadu, India

Dr. Barathiselvan V

Lecturer, Department of

Orthopaedics, Rajah Muthiah

Medical College, Chidambaram,

Tamil Nadu, India

\section{Dr. Balamurugavel PS}

Lecturer, Department of

Orthopaedics, Rajah Muthiah

Medical College, Chidambaram,

Tamil Nadu, India

\section{Dr. Parasuraman}

Assistant Professor, Department of Orthopaedics, Rajah Muthiah Medical College, Chidambaram,

Tamil Nadu, India

Dr. Ravikrishna R

Senior Resident, Department of Orthopaedics, Rajah Muthiah

Medical College, Chidambaram, Tamil Nadu, India
Corresponding Author: Dr. Neelakrishnan $R$

Professor and Head, Department of Orthopaedics, Rajah Muthiah Medical College, Chidambaram, Tamil Nadu, India

\section{Comparison of outcome of tibial plafond fractures managed by external fixation with minimal fixation versus open reduction and internal fixation}

\author{
Dr. Prabhu S, Dr. Neelakrishnan R, Dr. Barathiselvan V, Dr. \\ Balamurugavel PS, Dr. Parasuraman and Dr. Ravikrishna R
}

DOI: https://doi.org/10.22271/ortho.2020.v6.i4i.2389

\begin{abstract}
Background: Pilon fractures are distal tibial intra-articular fractures with proximal extensions and often associated with extensive soft tissue damages. There is no level 1 treatment for tibial pilon fractures. Some experts advice bridging external fixators combined with limited fixation to minimize the soft tissue injuries and some authors advocate ORIF technique to prevent articular incongruence. Attaining anatomical reconstruction of the joint, restoring the tibial alignment, and fracture stabilization are the methods to achieve an optimal outcome. Fracture morphology and soft tissue injuries must taken into serious consideration for the pre-operative planning as they are the frequent cause for complications. External fixation combined with limited fixation or open reduction and internal fixation are different modes of management. LFWEF have the advantage of preserving the vascularity of the fracture and
\end{abstract} minimizes the soft tissue compromise but pin tract infection is the common complication.

Aim: The aim of the study was to compare the outcome of external fixation combined with limited fixation vs open reduction and internal fixation in restoring and fixing the articular surface for either open or closed tibial pilon fractures.

Patients and Methods: This study includes 15 patients with pilon fractures of Reudi Allgower type I, II, III, treated by either LFWEF or ORIF. Their clinical and functional outcome and radiological outcome were compared. They were evaluated for the degree of involvement of articular surface and the condition of the soft tissue envelop around the fracture for surgeries. Before surgery, all patients were evaluated clinically and radiologically. All patients were followed up till the achievement of fracture union and soft tissue healing. Evaluation was done based on anatomical articular reduction, achievement of union in time and function of the ankle joint using AOFAS ankle scoring system.

Results: The mean average time of union was 17 weeks (range 12-21 weeks) for all the fractures after fixation. 12/15 of cases had near anatomical restoration of the articular surface, representing $80 \%$ of cases. Acceptable alignment seen in 14/15 cases cases, representing $93.6 \%$ of cases in this study. In my study two cases showed limitation of range of movements (2/15 cases), representing $13.3 \%$ of cases. Only one case showed deep soft tissue infection (1/15) representing $6.6 \%$. The results of external fixation combined with limited fixation is more effective in terms of soft tissue management, early union and superficial or deep infections than ORIF.

Conclusion: LFWEF have the advantages of minimizing soft tissue insult and preserving the vascularity and hematoma thereby reducing fracture union time but the disadvantages are pin tract infection. ORIF was associated with higher risk of post operative complication with respect to superficial or deep infection, delayed union and non union than external fixation with limited fixation.

Keywords: Vascularity and hematoma, external fixator, pilon fractures, soft tissue infection

\section{Introduction}

A pilon fracture is a fracture that involves articular surface of the distal tibia with proximal extension from the ankle articular surface. Pilon fractures are due to rotational or axial-loading forces as a result of high-energy trauma. Plafond means ceiling or dome. The Talus has tibial plafond as a dome or ceiling and articulates with the distal tibia with its smooth surface. Destot described pilon fractures as 'explosive injuries'. Rotational force may produce spiral fractures it may be extra-articular or intra-articular. Usually rotational force produce less soft tissue injuries whereas axial compressive force produce intra-articular fractures with more 
soft tissue injuries. Unopposed plantar flexion produce fracture at the posterior articular surface. On the otherhand unopposed dorsiflexion produce fracture at the anterior part of the tibial articular surface. But on neutral position, there will be ' $y$ ' shaped separation of anterior and posterior fragments due to central joint impaction. These fractures are challenging to treat because of their subcutaneous location and minimal soft tissues. Managing comminuted fracture, displaced fragments, fracture at metaphyseal region are always complex to treat.

Several surgical methods have been suggested for the treatment of pilon fractures which includes external fixation with limited fixation, open reduction and internal fixation with plates and screws. In these days many surgeons started treating pilon fracture with external fixation combined with limited fixation and leaving the fixators in place until bone union achieved. Pin tract infections are most likely to occur as a complication in external fixation.

\section{Patients and methods}

This prospective clinical study was carried out from October 2018 to October 2020 after ethical committee approval in the Orthopaedic Department of Rajah Muthaiah Medical College and Hospital, and included 15 patients with tibial pilon fractures. Their ages ranged from 20 to 64 years (mean 32.8 years). Ten patients (66.6\% of cases) had closed pilon fractures and the remaining five (33.3\% of cases) presented with open pilon fractures. Patients differed with fracture pattern and the severity of soft tissue injury, either closed or open fractures. Fractures classified according to the Ruedi and Allgower fracture classification. Open fractures were classified under the Gustilo and Anderson classification, whereas closed fractures classified with Tscherne classifications. The ankle and foot were clinically examined for any neuro vacular deficit, or any impending compartment syndrome. Dorsalis pedis pulse to be checked to know about the circulary status of the foot, if the pulse is uncertain Doppler study should be utilized. The examination includes antero-posterior, mortise, and lateral views are the standard radiographic views and must include the entire tibia and fibula and the foot. CT with 3D reconstruction was carried out to know about the fracture line, fracture pattern and the number of fragments and to assess the articular surface. Antiseptic Wound debridement, pulsatile lavaging were done in the casualty room and antibiotics started immediately for open fractures. All surgeries were done under spinal anesthesia. After surgery, patients with external fixators and limited fixation were started on active range of motion (ROM) of the ankle joint with foot elevation during rest to avoid congestion and edema. Daily dressings to be done for open pilon fracture using normal saline solution and hydrogen peroxide for Schanz screws to prevent pin tract infection. Absolute non weight-bearing until the early callus formation was adviced, early callus formation coincided with the soft tissue healing in most of the open cases. After union was achieved, without anesthesia external fixators were removed in the clinic and a protective cast or brace was applied until complete union. Pin tract infections was decribed by checketts -otterburns classification.

\section{Sample size}

Fifteen patients were selected for this study. Of those 6 cases are type I, 4 cases are type II and 5 cases are type III according to RA classification.

Table 1: Different patient groups

\begin{tabular}{|c|c|c|}
\hline Gustilo and Anderson classification & Tscherne classification for closed fractures & Ruedi and Allgower fracture classification \\
\hline Type I: no cases & Grade I: 3/10 cases & Type I: $6 / 15$ cases \\
\hline Type II: $2 / 5$ cases & Grade II:7 /10 cases & Type II: $4 / 15$ cases \\
\hline Type III: $3 / 5$ cases & Grade III: no cases & Type III: $5 / 15$ cases \\
\hline
\end{tabular}

\section{Patient selection and randomization}

Fifteen patients were selected for the study. Patients of aged 18 years or more, with a recent (less than 3 weeks) closed or open intraarticular distal tibial fractures of Rüedi and Allgower type I, II and III were included in this study. Patient with pathological fractures, associated spinal injuries / head injuries/chest injuries, bleeding disorders, associated fractures of other bones of ispilateral limb (except fibula) were excluded.

\section{Statistical analysis}

Data were coded, entered and processed. The cut-off value for significance was set at $\mathrm{p}<0.05$ student ' $\mathrm{t}$ ' test was used to assess the statistical significance of difference between two population.

Table 2: Demographic data

\begin{tabular}{|c|c|c|}
\hline Parameter & LFWEF & ORIF \\
\hline Number & 6 & 9 \\
\hline Mean age & 33 & 31 \\
\hline Gender M:F & $4: 2$ & $6: 3$ \\
\hline Days to operation & $1-2$ & $9-12$ \\
\hline Follow up (month) & $16-20$ & $18-24$ \\
\hline Operative time (minute) & 90 & 120 \\
\hline Healing time (days) & 95 & 125 \\
\hline
\end{tabular}


Case 1.
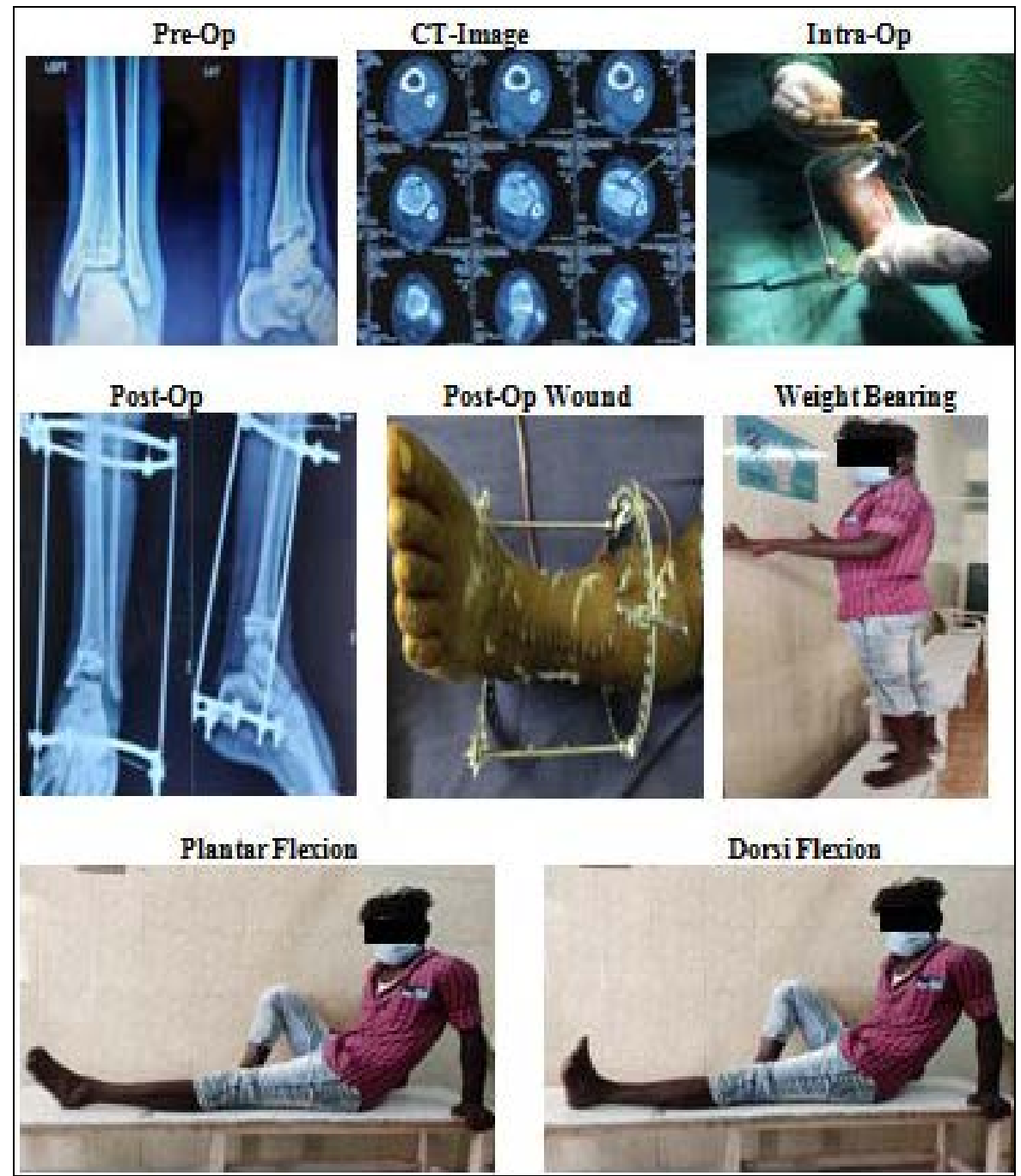

Case 2.
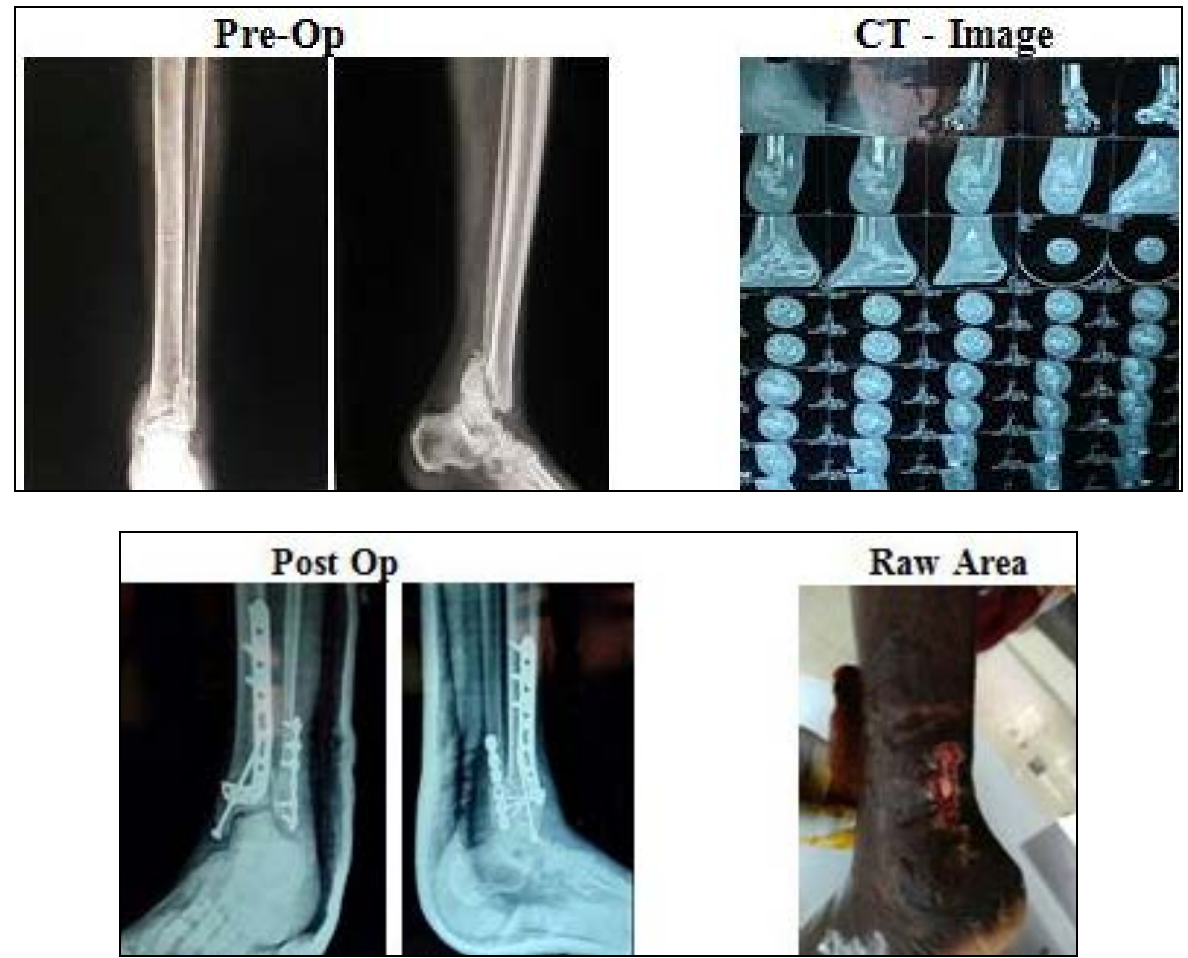

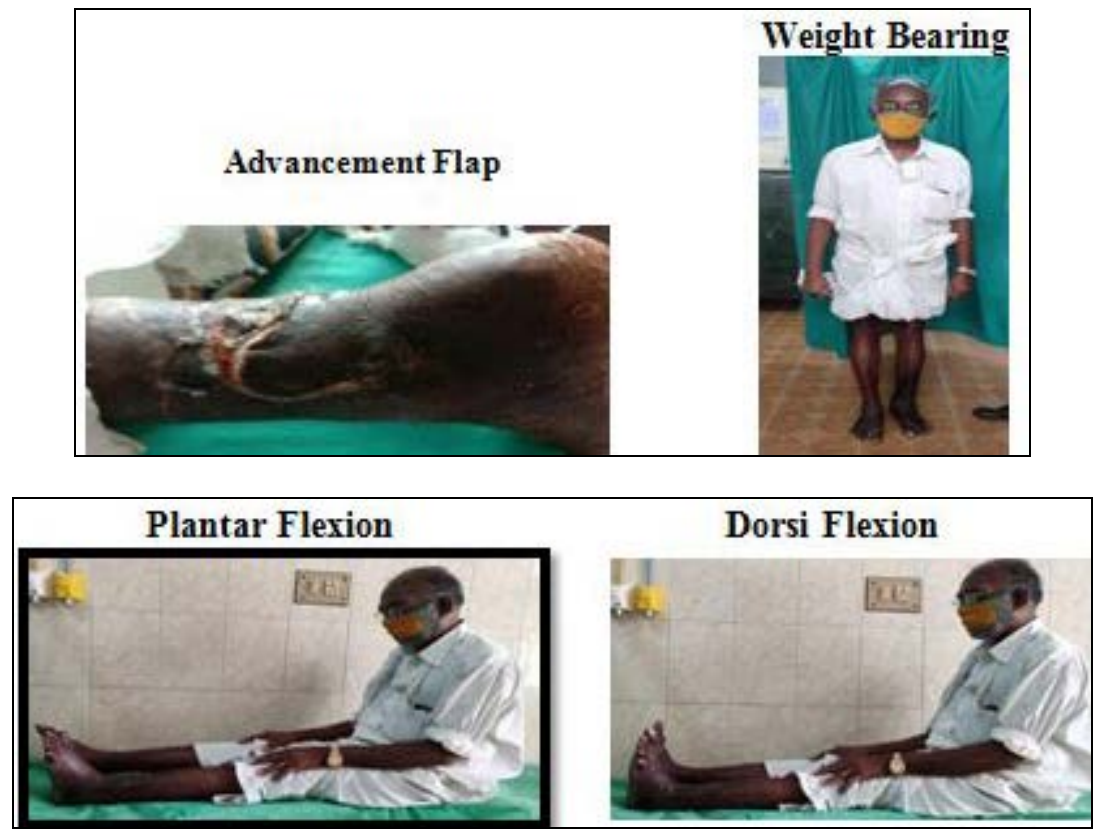

\section{Results}

The average time of 24 months (range 10-19 months) were the following period for all the patients, results were analysed both clinically and radiologically. The average time for all the fracture union was achieved in 17 weeks (range 12-21 weeks). Patient with good anatomical articular reduction are completely united in 12 of the 15 cases, which represents $80 \%$ of the studied group. The remaining three cases had anatomical reduction beyond the acceptable criteria which represents $20 \%$. The varus-valgus angulation of less than $5^{\circ}$ and anteroposterior angulation less than $10^{\circ}$ and shortening of less than $15 \mathrm{~mm}$ are acceptable. Ankle joint functions are evaluated by IOWA ankle ranking system.

In our study 12 of the 15 patients showed excellent results (score $\geq 90$ ), representing $80 \%$ of the studied population, and good results (score 80-90) were reported in one case, representing $6.7 \%$ of the population. Two cases had fair results (score 70-79), representing $13.3 \%$, and no patients with poor results (score $<70$ ) were reported in this study. Thus, satisfactory results (excellent and good results) were obtained in 13 cases, representing $86.7 \%$ of the studied group. No cases developed deep infection even in open cases. No cases required skin grafts or flaps. Results were analyzed from the view of fracture morphology, either open or closed, and the type of external fixator applied, either spanning the ankle or not. Non-spanning external fixators were used for closed pilon fracture with minimal articular comminution that showed good results which allows early weight-bearing and early active ROM of the ankle and spanning external fixator were used for severely comminuted open pilon fractures which showed less favorable results than the previous group regarding the early range of movements, malunion and Angulation.

Table 3: Clinical results

\begin{tabular}{|c|c|c|c|}
\hline Parameter & LFWEF & ORIF & $\boldsymbol{p}$ value \\
\hline AOFAS & 86.7 & 82.3 & 0.3 \\
\hline Pin tract infection & 1 & 0 & \\
\hline Delayed union & 0 & 1 & \\
\hline Non union & 0 & 0 & \\
\hline Arthritis & 0 & 1 & \\
\hline
\end{tabular}

AOFAS: American Orthopaedic Foot and Ankle Society; LFWEF: limited fixation with external fixation; ORIF: Open Reduction and Internal Fixation

\section{Complications}

Managing pilon fractures is a complex one that can occur at any time either intra-operatively or in the early or late post operative period. Pre operative planning and a meticulous surgical technique is much needed in pilon fracture as perioperative complications like inadequate reduction, inadequate fixation, and hardware penetration into the articular joint, can be minimized. Open injuries are highly potential to have deep infection as early complication and stiffness, post-traumatic arthritis are late complications, all of which decided by the severity of initial injury and the accuracy of articular reduction. In this study, one patient developed deformity in the form of varus angulation deformity more than $15^{\circ}$ because patient had severe tibial metaphyseal comminution, articular impaction with soft tissue injury on day of admission. This patient had limited range of movements and was put on physiotherapy and improved later.

\section{Discussion}

Distal tibial fractures includes a wide variety of lesions from simple metaphyseal and diaphyseal fractures to articular impaction and severe soft tissue injuries. When the trauma energy increases, there is a higher rate for complex distal tibial fractures with soft tissue injuries, and a higher rate for complications in future to occur. The main goal of treatment of tibial pilon fractures are to realign the fracture, maintaining the limb length axis, restoring the joint surfaces, and recreation of limb alignment. Nowadays advanced surgical techniques, implants and approaches have been proposed with a wide range of results. Though ORIF technique were used initially now it is known that this technique increases the complication rate so various methods of external fixation have been evolved. Limited fixation with external fixation reduce fractures by ligamentotaxis with minimal interference of soft tissues. It is an indirect method of reducing the fracture and providing points of fixation away from the injury area so it was accepted by all to manage pilon fractures with poor soft 
tissue injuries. It is believed that using Ilizarov system improves the technique of indirect reduction, early mobilization and periodical correction of deformities. In our study average union time was 17 wks. Cases with external fixators shows less aggression to the soft tissues and this factor determines the satisfactory union to achieve at a faster rate. So limited fixation with external fixation allows early mobilization and weight bearing. Aggressive surgical approaches and systems that are too rigid might favour to the development of non-union. There was a lower rate of reoperations noticed in LFWEF. Using thin wire external fixation decreases the rates of infection, whereas higher rate of infections were noticed in ORIF as the skin vascularity might be compromised by the open approach.

Luis et al. ${ }^{[1]}$ noticed that the average time required for surgery to weight bearing and fracture to radiological union was less in external fixator group. Regi Samuel [2] reported good functional outcome in type II and type III fractures treated with external fixation and bone grafting. Rayan [3] noticed equally effective results in view of fracture union and functional outcome and complication rate in both the groups. Xueliang Cui ${ }^{[4]}$ reported insignificant differences in delayed union, malunion, deep infections and arthritis in both the groups. Rizk Ahmed [5] concluded eternal fixation with limited fixation in treating tibial plafond fractures have advantages in minimizing soft tissue injuries and preserves vascularity and there-by shortening the fracture union time and the need for bone grafting were reduced. Shota Harada ${ }^{6]}$ proposed using circular frame external fixator shows good results in severely comminuted open pilon fractures. Justin E Richards ${ }^{[7]}$ demonstrated faster union and good functional outcomes of ankle function in patients treated with ORIF. In our study lesser union time and early ankle functional recovery noticed in LFWEF group. Sean et al. ${ }^{\left[{ }^{8]}\right.}$ found that re-operation required in open pilon fractures who were treated with ORIF, but in our study patient with external fixator group never had any re-do in surgical procedures. Chowdhry and Porter [9] reported pin tract infection and inadequate reduction in few cases treated with external fixator, whereas in our study only one case had pin tract infection as sterile pin tract dressings done on a regular basis for all the cases. Cesar ${ }^{\text {[10] }}$ reported development of deep infection in $23.2 \%$ of open fractures vs $11.3 \%$ in closed fracture. In our study only one deep infection occurred in patient treated with ORIF. Ruedi [11] believed that open reduction and internal fixation as the ideal technique for the severely comminuted fracture provided with perfect anatomical reconstruction and stable internal fixation. Pouya Mafi ${ }^{[12]}$ evaluated the merits of external fixator in minimizing the complication. He placed fine wire Ilizarov frame far to the site of injury and allowed early weight bearing. Lisa ${ }^{[13]}$ proposed no- touch approach to minimize soft tissue complications by using external fixator rather than ORIF. Mitkovic ${ }^{[14]}$ showed $71 \%$ excellent and good results by treating comminuted pilon fracture with external fixator and also had no cases with non union or deep infection.

\section{Conclusion}

To achieve an optimal outcome reconstructing the articular surface anatomically, restoring the fibular length and tibial alignment are the main elements

Table 4: Merits of LFWEF over ORIF

\begin{tabular}{|c|c|}
\hline LFWEF & ORIF \\
\hline - Rapidly performed & \\
- working far from the site of injury. & - Secondary insults to soft tissues. \\
- Prevents secondary soft tissue insults. & - Soft tissue retraction. \\
- Easy access for the wound dressing. & - Can check tissue viability. \\
- Less surgery time. & - Consumes more time. \\
- Fracture unites faster by preserving & - Joint stiffness \\
haematoma. & - Deep infections are common. \\
- Early mobilization and weight bearing. & \\
-periodical correction of deformities & \\
\hline
\end{tabular}

Stripping muscles of the bone soft tissue retraction are the factors which contribute for delayed healing, delayed union / non-union of the fracture in ORIF. Using tornique for a longer time also contribute to soft tissue complications. The severe deep infection may require free flap in ORIF but not so in external fixation.The study concluded that with adequate articular reduction, ORIF and LFWEF can achieve union in time but by means of preserving vacularity and reducing soft tissue insult, LFWEF group found to have union rates at a faster pace than ORIF group.

\section{References}

1. Luis Cisneros, Natera et al. Comparison of outcome of tibial plafond fractures managed by hybrid external fixation versus two-stage management with final plate fixation. Indian Journal of Orthopaedics 2016;50(2):12330. doi:10.4103/0019-5413.177577

2. Reji Varghese, Shajimon Samuel. Functional outcome of JESS fixation and bone grafting in distal tibial plafondpilon fracture: a prospective study. DOI: http://dx.doi.org/ 10.18203/issn.2455-
4510.IntJResOrthop20182742

3. Ahmed R, Ahmed K, Elmoatasem Elhussein M, et al. Open Reduction Internal Fixation versus External Fixation with Limited Internal Fixation for Displaced Comminuted Closed Pilon Fractures: A Randomised Prospective Study. Journal of Orthopaedics, Trauma and Rehabilitation 2018;24(1):84-89. doi:10.1016/j.jotr.2017.08.001

4. Cui X, Chen H, Rui Y, Niu Y, Li H. Two-stage open reduction and internal fixation versus limited internal fixation combined with external fixation: a meta-analysis of postoperative complications in patients with severe Pilon fractures. J Int Med Res. 2018;46(7):2525-2536.

5. Rizk AS, Singer MS, Al-Ashhab ME. Combined minimally invasive external and internal fixation in the treatment of pilon fractures. Egypt Orthop J [serial online] [cited 2020 Nov 10] 2014;49:259-65. Available from: http://www.eoj.eg.net/text.asp?2014/49/3/259/1481 98

6. Shota Harada Tsukasa Teramoto Motoyuki Takaki Tomohiko Asahara Narutaka Katoh Nobuyuki Takenaka 
Takashi Matsushita Ankle joint reconstruction by circular frame external fixator for a severely comminuted and contaminated open tibial pilon fracture (AO 43-C3.3). Case report https://doi.org/10.1016/j.tcr.2019.100234

7. Justin Richards E, Mark Magill, Marc Tressler A, Franklin Shuler D, Philip Kregor J, William Obremskey T. Southeast Fracture Consortium External fixation versus ORIF for distal intra-articular tibia fractures. DOI: 10.3928/01477447-20120525-25

8. Sean T, Campbell L. Henry Goodnough, Brett Salazar, Justin Lucas F, Michael J. Gardner How do pilon fractures heal? An analysis of dual plating and bridging callus formation. I:https://doi.org/10.1016/j.injury.2020.04.023

9. Chowdhry M, Porter K. The pilon fracture. Trauma. 2010;12(2):89-103. doi:10.1177/ 1460408609359874

10. Cesar Molina S, Stinner DJ, Fras AR, Evans JM. Risk factors of deep infection in operatively treated pilon fractures (AO/OTA: 43). J Orthop 2015;12(1):S7-S13. Published 2015 Feb 21. doi:10.1016/j.jor.2015.01.026

11. Rüedi T. Fractures of the lower end of the tibia into the ankle joint: results 9 years after open reduction and internal fixation. Injury 1973;5(2):130-4. doi: 10.1016/s0020-1383(73)80089-0. PMID: 4774764.

12. Pouya Mafi, James Stanley, Sandip Hindocha, Reza Mafi. Pilon Fracture: A Case Report of a 45-Year-Old Dental Technician.

13. Lisa Cannada K. The No-Touch Approach for Operative Treatment of Pilon Fractures to Minimize Soft Tissue Complications https://doi.org/10.3928/0147744720100826-16

14. Mitkovic MB, Bumbasirevic MZ, Lesic A, Golubovic Z. Dynamic external fixation of comminuted intra-articular fractures of the distal tibia (type $\mathrm{C}$ pilon fractures). ActaOrthop Belg. 2002; 68(5):508-14. PMID: 12584982. 